\title{
AURKA and BRCA2 expression highly correlate with prognosis of endometrioid ovarian carcinoma
}

\author{
Fan Yang ${ }^{1,2}$, Xiaoqing Guo ${ }^{1}$, Gong Yang ${ }^{1}$, Daniel G Rosen ${ }^{1}$ and Jinsong Liu ${ }^{1}$ \\ ${ }^{1}$ Department of Pathology, The University of Texas MD Anderson Cancer Center, Houston, TX, USA and \\ ${ }^{2}$ Department of Pathology, West China Second University Hospital, Sichuan University, Chengdu, \\ Sichuan, People's Republic of China
}

\begin{abstract}
Aurora kinase A (AURKA), a serine/threonine kinase, has been shown to regulate the cell cycle checkpoint and maintain genomic integrity. AURKA is overexpressed in various carcinomas. Breast cancer 2, early onset (BRCA2) has an important role in maintaining genomic stability and acts as a tumor suppressor. Our recent study suggested that AURKA regulates genomic instability and tumorigenesis through cell cycle dysregulation and suppression of BRCA2 expression. However, the expression of AURKA, BRCA2 and their clinical significance is unknown in endometrioid ovarian cancer. In this study, we determined AURKA and BRCA2 expression in endometrioid ovarian carcinoma and correlated them with clinicopathologic characteristics and patient survival. Immunohistochemical staining was performed in $\mathbf{5 1}$ primary endometrioid ovarian carcinoma tumor samples, using tissue microarray. We then analyzed the associations between AURKA and BRCA2 expression and clinical factors (tumor grade, disease stage, surgical type, clinical response, and relapse) and overall and disease-free survival durations. AURKA and BRCA2 expression were found in 48 and $29 \%$ of the samples, respectively. The results of Fisher's exact test suggested that AURKA expression was significantly associated with no family history of ovarian cancer $(P=0.03)$ and that BRCA2 expression was associated with early-stage disease $(P=0.03)$, low ascites incidence $(P=0.03)$, younger age $(<60)$ at diagnosis $(P=0.03)$, and low-grade tumors $(P<0.01)$. The nuclear BRCA2 score was negatively correlated with AURKA score $(P=0.019$, two-tailed Pearson correlation). A log-rank test demonstrated that AURKA expression was associated with shorter overall $(P=0.001)$ and disease-free $(P=0.009)$ survival durations, and that BRCA2 expression was associated with longer overall $(P=0.000)$ and disease-free $(P=0.002)$ durations. Patients with BRCA2-positive and AURKA-negative tumors had higher overall $(P=0.001)$ and disease-free $(P=0.001)$ survival rates than did patients with AURKA-positive and BRCA2-negative tumors. Our results demonstrate that a negative regulatory loop exists between AURKA and BRCA2 expression in the ovarian endometrioid carcinoma. AURKA expression is an unfavorable prognostic factor in patients with endometrioid ovarian cancer and BRCA2 is favorable, combination of these two markers may better predict the prognosis of patients with endometrioid ovarian carcinoma than individual marker alone.
\end{abstract}

Modern Pathology (2011) 24, 836-845; doi:10.1038/modpathol.2011.44; published online 25 March 2011

Keywords: AURKA; BRCA2; endometrioid ovarian carcinoma; prognostic factors

Endometrioid ovarian carcinoma accounts for $20-25 \%$ of ovarian carcinomas, surpassed in

Correspondence: Dr J Liu, Department of Pathology, Unit 085, The University of Texas MD Anderson Cancer Center, 1515 Holcombe Boulevard, Houston, TX 77030, USA.

E-mail: jliu@mdanderson.org

Received 6 October 2010; revised 13 December 2010; accepted 29 December 2010; published online 25 March 2011 frequency only by serous carcinoma. Endometrioid ovarian carcinoma is most common in women in their 50s (mean age at diagnosis, 56 years) and has a 5 -year survival rate of $40-52 \%$, with most patients presenting with early-stage disease. ${ }^{1}$ The coincidence of endometriosis and endometrioid ovarian carcinoma has been well documented in the medical literature, and the possibility of a direct association has been suggested in $30-40 \%$ of patients. ${ }^{2}$ 
The results of some studies have suggested that endometrioid ovarian carcinoma is associated with a better outcome than is serous carcinoma. ${ }^{3,4}$ Contrarily, another study showed no difference in prognosis when the clinical characteristics were similar. ${ }^{5}$ Despite its widespread use, the Gynecologic Oncology Group's endometrioid ovarian carcinoma grading system can be difficult to use because of its poor interobserver reproducibility and low association with prognosis. ${ }^{6-8}$ Therefore, it is important to identify prognostic indicators for endometrioid ovarian carcinoma.

Recent advances in molecular techniques have shed some light on the molecular events that lead to ovarian carcinoma. The serine/threonine kinase, aurora kinase A (AURKA), also called Aurora-2, BTAK, ARK1, and STK15, maintains cell division by regulating centrosome separation, bipolar spindle assembly, and chromosome segregation. ${ }^{9,10}$ However, AURKA overexpression also results in a defective spindle assembly checkpoint, allowing cells with abnormal chromosomal separation to enter anaphase, leading to aneuploidy and inducing genomic instability. ${ }^{11}$ Human AURKA is amplified and overexpressed in various carcinomas, including colorectal, ${ }^{12}$ bladder, ${ }^{13}$ pancreatic, ${ }^{14}$ gastric, ${ }^{15}$ and breast $^{16}$ cancers. AURKA overexpression can transform mouse NIH/3T3 cells by inducing centrosome amplification and aneuploidy. ${ }^{10}$ In ovarian cancer, AURKA activation or overexpression is found in cancer cell lines ${ }^{17,18}$ and tumor specimens, ${ }^{19,20}$ and is associated with poor prognosis in cancer patients. $^{20,21}$ The results of these studies indicate that AURKA has a key role in the pathogenesis of human cancers by maintaining genetic stability.

Breast cancer 2, early onset (BRCA2) has an important role in maintaining genomic stability and acts as a tumor suppressor. BRCA2 repairs the double-strand DNA break during the $S$ phase of the cell cycle, and participates in cytokinesis. The abnormalities in chromosome number seen in BRCA2-deficient cells are a direct consequence of BRCA2 dysfunction. Moreover, BRCA2's role in cytokinesis provides a mechanism for polyploidy. ${ }^{22}$ The results of a recent study suggested that AURKA regulates genomic instability and tumorigenesis through cell cycle dysregulation and BRCA2 suppression. This negative correlation between AURKA and BRCA2 has been found in multiple cancer types. ${ }^{23}$

AURKA and BRCA2 expression and their clinical significance are unknown in endometrioid ovarian carcinoma. We hypothesize that their expression is correlated with clinical outcome. Therefore, in this retrospective study, we evaluated the association between AURKA and BRCA2 expression and clinical pathologic factors (including tumor grade, disease stage, surgical type, clinical response, and relapse), and overall survival and disease-free survival rates in endometrioid ovarian carcinoma patients.

\section{Materials and methods}

\section{Patients and Clinicopathologic Data}

We identified all primary endometrioid ovarian carcinoma patients who had undergone initial surgery at The University of Texas MD Anderson Cancer Center (Houston, TX) from 1990 to 2006, by reviewing electronic charts. Only patients with pure endometrioid ovarian carcinoma were included in the final analysis; those with mixed-type carcinomas with an endometrioid component were excluded. Patient charts were reviewed to exclude those cases that may have represented other primary tumor sites, such as the colon. All of the cases were reviewed independently at least by two pathologists (JL, FY), with the same cohort that we used in our previous study. ${ }^{8}$ Immunohistochemical staining had been performed, if necessary, at diagnosis to exclude other type of tumors and other primary tumor sites, WT-1 is one of markers we used to differentiate high grade endometrioid ovarian carcinoma and high grade serous carcinomas. Patients' follow-up information was updated through March 2010 by reviewing medical records. Demographic and survival data were entered into a comprehensive database created with Microsoft Access (version 2007; Microsoft, Redmond, WA, USA). Histopathologic diagnoses were based on World Health Organization criteria, tumor grades were based on Gynecologic Oncology Group criteria, and disease stages were assessed according to the International Federation of Gynecology and Obstetrics system. ${ }^{24}$ Overall survival durations were computed as the time from the date of first biopsy to the date of death or last followup, whichever occurred first. Patients alive at the last follow-up date were censored. Disease-free survival durations were computed as the time from the date of first biopsy to the date of recurrence, death, or last follow-up, whichever occurred first. Patients alive with no recurrence at last follow-up were censored. Patients in the progressive disease group were those in whom no disease remission had been observed after treatment. Patients in the relapsed disease group were those in whom disease remission had been clinically documented. The extent of cytoreduction was defined as optimal if the largest diameter of any residual lesion from surgery had been smaller than $1 \mathrm{~cm}$, or suboptimal if larger than $1 \mathrm{~cm} .{ }^{25,26}$ The use of tissue blocks and the chart review were approved by the institutional review board of MD Anderson.

\section{Construction of Tissue Microarrays}

Tissue blocks had been stored under ambient conditions at approximately $24^{\circ} \mathrm{C}$. A pathologist reviewed hematoxylin- and eosin-stained sections to select representative areas of tumor from which cores could be acquired for microarray analysis. Tissue microarray blocks were constructed as 
described earlier, ${ }^{27}$ with a precision instrument (Beecher Instruments, Silver Spring, MD, USA) that uses two separate core needles for punching the donor and recipient blocks and a micrometerprecise coordinate system for assembling tissue samples on a block. For each case, two replicate of 1-mm core-diameter samples were collected and placed on separate recipient blocks. The final tissue microarray consisted of one block containing duplicates of 51 spots. All samples were spaced $0.5 \mathrm{~mm}$ apart. Sections of $5 \mu \mathrm{m}$ were obtained from the microarray and stained with hematoxylin and eosin to confirm the presence of tumors and assess tumorhistologic characteristics. Tumor samples were randomly arranged on blocks.

Sample tracking was based on coordinate positions for each tissue spot in the tissue microarray block; the spots were transferred onto tissue microarray slides for staining. This sample tracking system was linked to a Microsoft Access database, containing demographic, clinicopathologic, and survival data for patients for whom samples were available, thereby allowing us to rapidly determine associations between histologic data and clinical features. The arrays were read according to the given tissue microarray map, each core was scored, and the results are presented as the mean of the two replicate core samples. Cases in which no tumor had been found or no cores were available were excluded from the final data analysis.

\section{Immunohistochemical Analysis}

Tissue microarray slides were treated and stained according to a previously published method. ${ }^{27}$ In brief, slides were deparaffinized in xylene and rehydrated in a graded series of ethanol, and sections were subjected to antigen retrieval by being boiled in $0.01 \mathrm{M}$ sodium citrate buffer $(\mathrm{pH}$ 6.0) in a microwave oven for $10 \mathrm{~min}$. After blocking the endogenous peroxidase activity and the nonspecific protein binding with $0.3 \%$ hydrogen peroxide and $1.5 \%$ normal goat serum, respectively, we incubated the sections overnight with primary antibodies at $4{ }^{\circ} \mathrm{C}$ in a humid chamber. The primary antibody against AURKA (GTX13824, monoclonal antibody, Genetax) or BRCA2 (MAB2476, monoclonal antibody, R\&D Systems) was applied at a dilution of $1: 200$ or $1: 100$, respectively, at $4^{\circ} \mathrm{C}$ in a humid chamber. A biotin-labeled secondary antibody (Universal Goat Link, Biocare Medical) was added for $15 \mathrm{~min}$, followed by horseradish peroxidase (Biocare Medical) for $15 \mathrm{~min}$. Tissues were then stained for 5 min with 3,3'-diaminobenzidine (Biocare Medical). Sections were lightly counterstained with hematoxylin. The primary antibody was replaced with $1 \times$ phosphate-buffered saline as a negative control. Staining intensity for immunohistochemical localization of BRCA2 and AURKA was independently determined by two pathologists
(F.Y. and J.L.) in a blinded manner. BRCA2 expression was scored for the number of cells with nuclear expression, whereas AURKA was scored for both cytoplasmic and nuclear staining. The pattern chosen for the analysis is the most consistent with previously published cell biology results; BRCA2 predominantly functions in the nucleus, whereas AURKA, a kinase, predominantly functions in the cytoplasm, although nuclear subcellular localization has been found in ovarian cancer cells. ${ }^{23}$ Thus, cores in which $<5 \%$ of cells were BRCA2 (nucleus) and AURKA (cytoplasm and nucleus) positive were considered negative (a score of 0 ), $5-20 \%$ were scored as $1,20-50 \%$ were scored as 2 , and $>50 \%$ were scored as 3 . The cases, that showed significant discrepancies in the staining of their two replicate core samples after repeated staining, were excluded from this study.

\section{Statistical Analysis}

Fisher's exact test and a logistic regression analysis were performed to evaluate the association between AURKA and BRCA2 with clinical factors. The Kaplan-Meier method was used to estimate the probability of overall and disease-free survival, and the log-rank test was used to compare overall or disease-free survival between comparison groups. Multivariate Cox proportional hazards regression models were fitted to determine the significant factors associated with overall and disease-free survival, and assess the association between AUR$\mathrm{KA}$ and BRCA2 expression and overall and diseasefree survival after adjusting for the effects of other clinical factors. The relationships between BRCA2 and AURKA expression were analyzed by Pearson's correlation coefficient. Results were considered statistically significant at the $P<0.05$ level. SPSS 17.0 software (SPSS, Chicago, IL, USA) was used for the statistical analysis.

\section{Results}

\section{Patient Characteristics}

A total of 51 patients were included in this study, with a median age of 58 years (range, 22-83 years). The median overall survival duration was 6.7 years (95\% confidence interval (CI): 2.1-11.4 years), and the overall survival rates were $79 \%$ (95\% CI: $0.73-$ 0.85 ) at 3 years, $68 \%$ (95\% CI: $0.61-0.74)$ at 5 years, and $55 \%(95 \%$ CI: $0.47-0.63)$ at 10 years. The median follow-up interval was 11.0 years, with a 95\% CI of 8.9-13.1 years.

\section{AURKA, BRCA2 Expression, and Localization}

Diffuse cytoplasmic and nuclear staining for AURKA and BRCA2 with moderate intensity were observed in different proportions of tumor cells. 
BRCA2
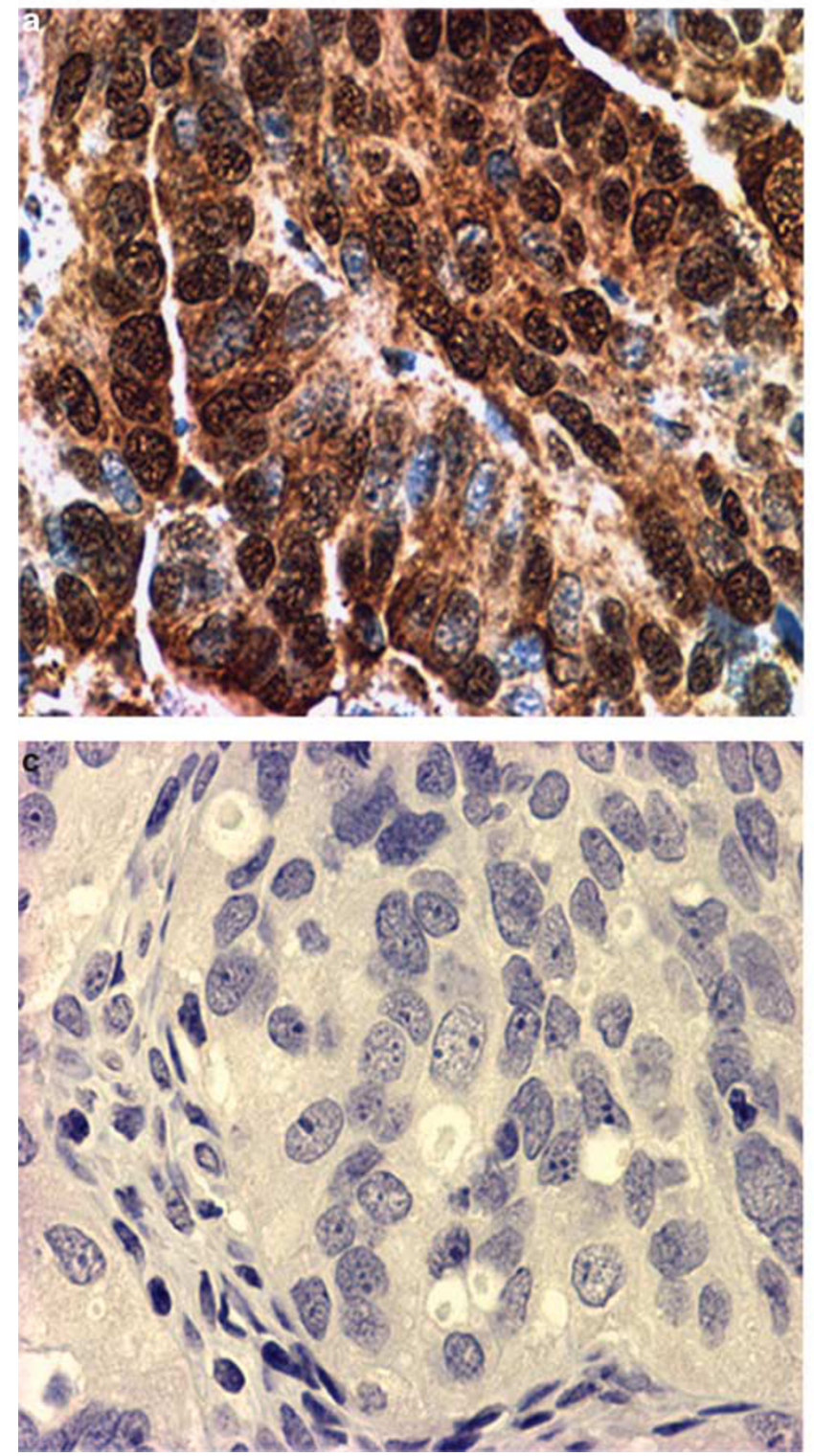
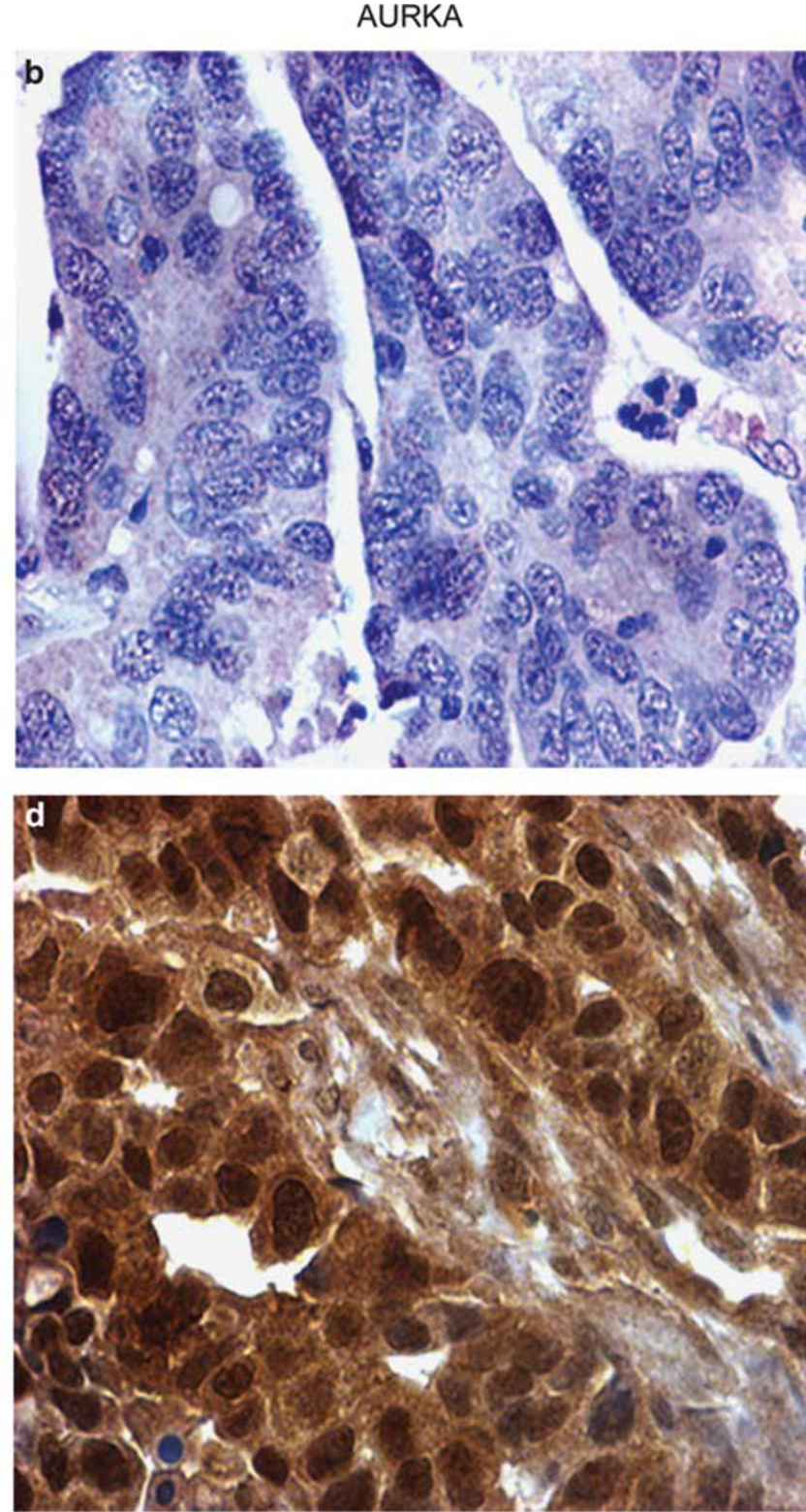

Figure 1 BRCA2 and AURKA expression in endometrioid ovarian carcinoma. (a) Endometrioid ovarian carcinoma cells show nuclear staining for BRCA2 $(\times 400)$. (b) AURKA-negative staining in endometrioid ovarian carcinoma ( $\times 400)$. (c) BRCA2-negative staining in endometrioid ovarian carcinoma $(\times 400)$. (d) Diffuse positive staining for AURKA in endometrioid ovarian carcinoma $(\times 400)$.

BRCA2 nuclear expression was scored and subjected to statistical analysis, whereas AURKA was scored for both cytoplasmic and nuclear staining. The percentage of positive cancer cells varied from $<5$ to $>50 \%$ in our patient population (Figure 1).

\section{Correlation between AURKA and BRCA2 Expression and Clinicopathologic Variables}

The tumor microarray immunohistochemical results, organized according to patients' clinicopathologic characteristics, are shown in Table 1. A total of 51 cases were included in this study after two cases excluded, because of significant discrepancies in the staining between their two replicate core samples. No cells expressed AURKA (score $=0$ ) in 22 patients (52\%), 5-20\% expressed AURKA in 18 patients ( $43 \%$; score $=1)$, and $20-50 \%$ expressed AURKA in two patients $(5 \%$; score $=2)$. No patients had a score of 3 . Nine cases for which no tumor was found or no cores were available for AURKA staining were excluded from the final data analysis. No cells expressed BRCA2 $($ score $=0)$ in 36 patients $(71 \%)$, $5-20 \%$ expressed BRCA2 in 5 patients $(10 \%$; score $=1), 20-50 \%$ expressed BRCA2 in 7 patients $(14 \%$; score $=2)$, and $>50 \%$ expressed BRCA2 in 3 patients $(5 \%$; score $=3)$.

The nuclear BRCA2 score was negatively correlated with AURKA total score $(P=0.019$, two-tailed 
Table 1 Correlation between AURKA and BRCA2 expression and clinicopathological characteristics

\begin{tabular}{|c|c|c|c|c|c|c|}
\hline \multirow[t]{2}{*}{ Characteristic } & \multicolumn{6}{|c|}{ No. of patients (\%) } \\
\hline & AURKA negative & AURKA positive & Total & BRCA2 negative & BRCA2 positive & Tota \\
\hline \multicolumn{7}{|l|}{ FIGO disease stage } \\
\hline Stage I & $5(45)$ & $6(55)$ & 11 & $8(67)$ & $4(33)$ & 12 \\
\hline Stage II & $3(50)$ & $3(50)$ & 6 & $4(36)$ & $7(64)$ & 11 \\
\hline Stage III & $12(67)$ & $6(33)$ & 18 & $18(86)$ & $3(14)$ & 21 \\
\hline Stage IV & $2(29)$ & $5(71)$ & 7 & $6(86)$ & $1(14)$ & 7 \\
\hline$P$-value & 0.36 & & & 0.03 & & \\
\hline \multicolumn{7}{|l|}{ Family history } \\
\hline Yes & $14(56)$ & $11(44)$ & 25 & $14(61)$ & $9(39)$ & 23 \\
\hline No & 2 (18) & $9(82)$ & 11 & $20(77)$ & $6(23)$ & 26 \\
\hline Unknown & & & 6 & & & 2 \\
\hline$P$-value ${ }^{\mathrm{a}}$ & 0.03 & & & 0.23 & & \\
\hline \multicolumn{7}{|l|}{ Age at diagnosis } \\
\hline$\geq 60$ & $10(56)$ & $8(44)$ & 18 & $20(83)$ & $4(17)$ & 24 \\
\hline$<60$ & $12(50)$ & $12(50)$ & 24 & $16(59)$ & $11(41)$ & 27 \\
\hline$P$-value ${ }^{a}$ & 0.53 & & & 0.03 & & \\
\hline \multicolumn{7}{|l|}{ Relapse } \\
\hline Yes & $9(64)$ & $5(36)$ & 14 & $15(75)$ & $5(25)$ & 20 \\
\hline Progressive disease & $2(40)$ & $3(60)$ & 5 & $6(100)$ & 0 & 6 \\
\hline No & $9(43)$ & $12(57)$ & 21 & $11(52)$ & $10(48)$ & 21 \\
\hline Unknown & & & 2 & & & 4 \\
\hline$P$-value ${ }^{\mathrm{a}}$ & 0.46 & & & 0.05 & & \\
\hline \multicolumn{7}{|l|}{ Type of surgery } \\
\hline Optimal & $15(45)$ & $18(55)$ & 33 & $26(65)$ & $14(35)$ & 40 \\
\hline Suboptimal & $5(71)$ & $2(29)$ & 7 & 7 (87) & 1 (13) & 8 \\
\hline Unknown & & & 2 & & & 3 \\
\hline$P$-value ${ }^{\mathrm{a}}$ & 0.09 & & & 0.23 & & \\
\hline \multicolumn{7}{|l|}{ Clinical response } \\
\hline Complete & $14(52)$ & $13(48)$ & 27 & $22(67)$ & $11(33)$ & 33 \\
\hline Partial & $2(67)$ & 1 (33) & 3 & 4 (100) & 0 & 4 \\
\hline None & $2(67)$ & $1(33)$ & 3 & $3(100)$ & 0 & 3 \\
\hline Unknown & & & 9 & & & 11 \\
\hline$P$-value ${ }^{\mathrm{a}}$ & 0.83 & & & 0.23 & & \\
\hline \multicolumn{7}{|l|}{ Ascites } \\
\hline No & $5(56)$ & $4(44)$ & 9 & $8(50)$ & $8(50)$ & 16 \\
\hline Yes & $9(45)$ & $11(55)$ & 20 & $16(80)$ & $4(20)$ & 20 \\
\hline Unknown & & & 13 & & & 15 \\
\hline$P$-value ${ }^{\mathrm{a}}$ & 0.43 & & & 0.03 & & \\
\hline \multicolumn{7}{|l|}{ CA125 (U/ml) } \\
\hline$<500$ & $2(25)$ & $6(75)$ & 8 & $6(55)$ & $5(45)$ & 11 \\
\hline$>500$ & $4(57)$ & $3(43)$ & 7 & $5(62)$ & $3(38)$ & 8 \\
\hline Unknown & & & 27 & & & 32 \\
\hline$P$-value ${ }^{\mathrm{a}}$ & 0.12 & & & 0.66 & & \\
\hline \multicolumn{7}{|l|}{ Grade } \\
\hline 1 & $3(50)$ & $3(50)$ & 6 & $4(57)$ & $3(43)$ & 7 \\
\hline 2 & $6(55)$ & $5(45)$ & 11 & $7(44)$ & $9(56)$ & 16 \\
\hline 3 & $13(52)$ & $12(48)$ & 25 & $25(89)$ & $3(11)$ & 28 \\
\hline$P$-value ${ }^{\mathrm{a}}$ & 0.91 & & & $<0.01$ & & \\
\hline
\end{tabular}

AUARK: aurora kinase A; BRCA2: breast cancer 2, early onset.

${ }^{\mathrm{a}} P$-values were calculated by Fisher's exact test.

Pearson correlation) in 42 cases in which BRCA2 and AURKA scores were available, as evidenced by the representative images that show positive BRCA2 and negative AURKA expression (Figure 1, upper panel), or negative BRCA2 and positive AURKA expression (Figure 1, lower panel).
BRCA2 expression ( $>5 \%$ of cells) was associated with early-stage disease $(P=0.03)$ (the cut-off for early vs late stage was I and II vs III and IV), low ascites incidence $(P=0.03)$, younger age $(<60)$ at diagnosis $(P=0.03)$, and low-grade tumors $(P<0.01)$ (the cut-off for low grade vs high grade tumor was 1 
and 2 vs 3). We found a trend towards earlier relapse in BRCA2-negative patients than in BRCA2-positive patients $(P=0.05)$. AURKA expression $(>5 \%$ of cells) was associated with no family history of breast or ovarian cancer $(P=0.03)$ (Table 1$)$.

The results of a multivariate logistic regression analysis suggested that BRCA2 was significantly associated with stage, ascites, and grade (Table 2). Patients with stage I endometrioid ovarian carcinoma were more likely to have BRCA2 expression than those with stage IV adenocarcinoma $(\mathrm{OR}=14.00$, $P=0.028$ ). Patients with grade 1 tumors or no ascites were more likely to have BRCA2 expression

Table 2 Multivariate analysis of factors associated with BRCA2 expression

\begin{tabular}{lcc}
\hline Variable & OR $(95 \%$ CI $)$ & $P^{\mathrm{a}}$ \\
\hline Stage & Reference & \\
IV & $14.00(1.33-147.43)$ & 0.028 \\
I & $0.35(0.07-1.71)$ & 0.195 \\
II & $0.23(0.021-2.59)$ & 0.236 \\
III & & \\
& Reference & \\
Ascites & $0.125(0.026-0.593)$ & 0.009 \\
Yes & & \\
No & Reference & 0.110 \\
Grade & $4.60(0.709-29.84)$ & 0.001 \\
3 & $13.80(3.12-61.14)$ & \\
2 & & \\
1 &
\end{tabular}

BRCA2: breast cancer 2, early onset; OR: odds ratio; $95 \%$ CI: $95 \%$ confidence interval.

${ }^{\mathrm{a}} P$-values were derived from multivariate logistic regression analysis.
$(\mathrm{OR}=13.80$ and 0.125 and $P=0.001$ and 0.009 , respectively).

\section{Association between AURKA and BRCA2 Expression and Overall and Disease-Free Survival}

The results of a multivariate Cox proportional hazards regression analysis indicated that stage, relapse, surgery type, clinical response, and BRCA2 and AURKA expression were significantly associated with overall and disease-free survival. Moreover, ascites was significantly associated with overall survival. Patients with late-stage (stage III and IV) disease, relapsed disease, suboptimal surgical type, ascites, no clinical response, and AURKA expression had a lower survival duration $(\mathrm{HR}=5.8$, $14.4,5.9,11.7$, and 0.3 and $P=0.006,0.001,0.001$, 0.014 , and 0.017 , respectively). BRCA2 expression was associated with a good outcome $(\mathrm{HR}=5.8$ $P=0.007$ ) (Tables 3 and 4).

The overall and disease-free survival rates at 3, 5, and 10 years are shown in relation to AURKA and BRCA2 expression in Table 5. At the time of this report, 22 of the 51 patients were alive without clinical evidence of ovarian carcinoma, eight were alive with ovarian carcinoma, 17 had died of ovarian carcinoma, three had died of other causes, and 1 had been lost to follow-up and was excluded from the overall and disease-free survival analyses. AURKA and BRCA2 expression were correlated with overall and disease-free survival duration. Patients with BRCA2-positive tumors had higher overall $(P=0.000)$ and disease-free $(P=0.002)$ survival rates

Table 3 Multivariate Cox proportional hazards model to estimate the association between covariate and disease free survival

\begin{tabular}{|c|c|c|c|c|}
\hline Variables & Estimate & s.e. & $P^{\mathrm{a}}$ & $H R(95 \% C I)$ \\
\hline Stage $(3,4$ vs 1,2$)$ & 1.75 & 0.64 & 0.006 & $5.8(1.6-20.3)$ \\
\hline Relapse (yes vs no) & 2.67 & 0.77 & 0.001 & $14.4(3.2-64.9)$ \\
\hline Surgery (suboptimal vs optimal) & 1.78 & 0.54 & 0.001 & $5.9(2.1-16.9)$ \\
\hline Clinical response (no vs yes) & 2.46 & 1.00 & 0.014 & $11.7(1.6-82.8)$ \\
\hline BRCA2 & -1.76 & 0.65 & 0.007 & $5.8(1.6-20.7)$ \\
\hline AURKA & 1.36 & 0.57 & 0.017 & $0.3(0.1-0.8)$ \\
\hline
\end{tabular}

AUARK: aurora kinase A; BRCA2: breast cancer 2, early onset; 95\% CI: 95\% confidence interval.

${ }^{\text {a } P}$ values were derived from multivariate Cox proportional hazards model analysis.

Table 4 Multivariate Cox proportional hazards model to estimate the association between covariate and overall survival

\begin{tabular}{|c|c|c|c|c|}
\hline Variables & Estimate & s.e. & $P^{\mathrm{a}}$ & $H R(95 \% C I)$ \\
\hline Stage $(3,4$ vs 1,2$)$ & 1.63 & 0.56 & 0.004 & $5.1(1.7-15.3)$ \\
\hline Relapse (yes vs no) & 2.04 & 0.64 & 0.001 & $7.7(2.2-26.9)$ \\
\hline Surgery (suboptimal vs optimal) & 2.06 & 0.53 & 0.000 & $7.9(2.8-22.4)$ \\
\hline Clinical response (yes vs no) & 2.40 & 0.85 & 0.005 & $11.1(2.1-58.4)$ \\
\hline Ascites (yes vs no) & 1.16 & 0.53 & 0.028 & $3.2(1.1-9.0)$ \\
\hline BRCA2 & -1.95 & 0.63 & 0.002 & $7.0(2.0-24.2)$ \\
\hline AURKA & 1.66 & 0.57 & 0.004 & $0.2(0.1-0.6)$ \\
\hline
\end{tabular}

AUARK: aurora kinase A; BRCA2: breast cancer 2, early onset; 95\% CI: 95\% confidence interval.

${ }^{\mathrm{a}} P$-values were derived from multivariate Cox proportional hazards model analysis. 
Table 5 Correlation between AURKA, BRCA2 expression and disease free and overall survival

\begin{tabular}{|c|c|c|c|c|c|c|}
\hline \multirow[t]{2}{*}{ Expression pattern } & \multirow{2}{*}{$\begin{array}{c}\text { No. of } \\
\text { patients }\end{array}$} & \multirow{2}{*}{$\begin{array}{l}\text { Median survival } \\
\text { years ( } 95 \% \text { CI) }\end{array}$} & \multicolumn{3}{|c|}{ Survival rate $(95 \%$ CI) } & \multirow[t]{2}{*}{$P^{\mathrm{a}}$} \\
\hline & & & 3 Year & 5 Year & 10 Year & \\
\hline \multicolumn{7}{|l|}{ AURKA } \\
\hline \multicolumn{7}{|l|}{ Disease free survival } \\
\hline- & 21 & $11.7(9.2-14.3)$ & $0.83(0.75-0.90)$ & $0.78(0.70-0.86)$ & $0.78(0.70-0.86)$ & 0.009 \\
\hline+ & 20 & $3.0(1.3-4.8)$ & $0.35(0.19-0.51)$ & $0.35(0.19-0.51)$ & - & \\
\hline \multicolumn{7}{|l|}{ Overall survival } \\
\hline- & 21 & $12.8(10.4-15.3)$ & $0.86(0.79-0.92)$ & $0.78(0.71-0.86)$ & $0.69(0.59-0.78)$ & 0.001 \\
\hline+ & 20 & $3.6(2.1-5.1)$ & $0.46(0.29-0.62)$ & $0.46(0.29-0.62)$ & - & \\
\hline \multicolumn{7}{|l|}{ BRCA2 } \\
\hline \multicolumn{7}{|l|}{ Disease free survival } \\
\hline- & 35 & $6.7(4.0-9.3)$ & $0.52(0.42-0.62)$ & $0.47(0.36-0.57)$ & $0.37(0.25-0.49)$ & 0.002 \\
\hline+ & 15 & $14.0(11.8-16.3)$ & $0.90(0.83-0.97)$ & $0.90(0.83-0.97)$ & $0.90(0.83-0.97)$ & \\
\hline \multicolumn{7}{|l|}{ Overall survival } \\
\hline- & 30 & $7.3(5.0-9.6)$ & $0.67(0.58-0.76)$ & $0.51(0.41-0.60)$ & $0.26(0.16-0.37)$ & 0.000 \\
\hline+ & 15 & $15.1(12.8-17.4)$ & $0.95(0.90-0.99)$ & $0.90(0.83-0.97)$ & $0.90(0.83-0.97)$ & \\
\hline \multicolumn{7}{|l|}{ AURKA, BRCA2 } \\
\hline \multicolumn{7}{|l|}{ Disease free survival } \\
\hline AURKA(-) BRCA2(+) & 14 & $13.6(10.8-16.5)$ & $0.93(0.86-0.99)$ & $0.93(0.86-0.99)$ & $0.93(0.86-0.99)$ & 0.001 \\
\hline AURKA(+) BRCA2(-) & 9 & $2.4(0.8-3.9)$ & $0.27(0.11-0.43)$ & $0.27(0.11-0.43)$ & - & \\
\hline \multicolumn{7}{|l|}{ Overall survival } \\
\hline AURKA(-) BRCA2(+) & 14 & $14.9(11.9-17.8)$ & $0.93(0.86-0.99)$ & $0.93(0.86-0.99)$ & $0.93(0.86-0.99)$ & 0.001 \\
\hline AURKA(+) BRCA2(-) & 9 & $3.0(1.8-4.2)$ & $0.40(0.22-0.56)$ & $0.26(0.10-0.42)$ & - & \\
\hline
\end{tabular}

AUARK: aurora kinase A; BRCA2: breast cancer 2, early onset; 95\% CI: 95\% confidence interval.

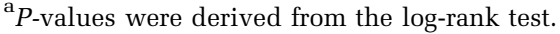

than did patients with BRCA2-negative tumors. In contrast, patients with AURKA-positive tumors had lower overall $(P=0.001)$ and disease-free $(P=0.009)$ survival rates than did patients with BRCA2negative tumors (Figure 2). In all, 14 cases were BRCA2 positive and AURKA negative, and nine were AURKA positive and BRCA2 negative. The results of our survival analysis suggested that patients with BRCA2-positive and AURKA-negative tumors had higher overall $(P=0.001)$ and diseasefree $(P=0.001)$ survival rates than did patients with AURKA-positive and BRCA2-negative tumors (Figure 2).

\section{Discussion}

In this study of well-characterized patients with long-term follow-up data, AURKA expression was found in $48 \%$ of 42 cases, and was correlated with no family history and poor survival; BRCA2 expression was observed in $29 \%$ of 51 cases, and was correlated with early-stage disease, a low ascites incidence, younger age $(<60$ years $)$ at diagnosis, low-grade tumors, and longer survival durations. BRCA2 expression was negatively correlated with AURKA expression in 42 cases. A recent study by our research group demonstrated that the positive percentage of AURKA and BRCA2 in serous ovarian carcinoma is about 40 and $15 \%$, respectively, ${ }^{23}$ which suggests that the percentage of BRCA2positive cells is higher in endometrioid ovarian carcinoma than in serous ovarian carcinoma. In our study, a multivariate Cox proportional hazards regression analysis revealed that early-stage disease, optimal surgical type, good clinical response, no ascites, BRCA2 expression, and no AURKA expression were strongly associated with longer overall and disease-free survival durations and were independent predictors of prognosis. Yang et $a l^{23}$ reported that in high-grade ovarian serous carcinoma, BRCA2 expression was associated with longer overall and disease-free survival durations, whereas AURKA expression was associated with shorter durations. AURKA and BRCA2 expression may be prognostic factors in ovarian carcinoma, not only in serous or endometrioid histotypes. This hypothesis should be investigated in other ovarian carcinoma histotypes.

The human AURKA gene is located in the 20q13 chromosome region and is involved in the G2-M checkpoint and mitosis commitment. ${ }^{28}$ AURKA interacts with p53 and BRCA1 to regulate the cell cycle checkpoint and maintain genomic integrity by phosphorylating p53 at Ser 215 and Ser 315 (8, 9), ${ }^{29,30}$ or BRCA1 at Ser $308(10) .{ }^{31,32}$ It is amplified and overexpressed in several malignant tumor types, including ovarian carcinomas. ${ }^{16,33}$ Our results demonstrate that AURKA expression in endometrioid ovarian carcinoma is associated with poor survival, which suggests that AURKA has a role in tumorigenesis. The tumor suppression function of BRCA2 is mediated by multiple processes, including suppression of cell proliferation ${ }^{34}$ and 

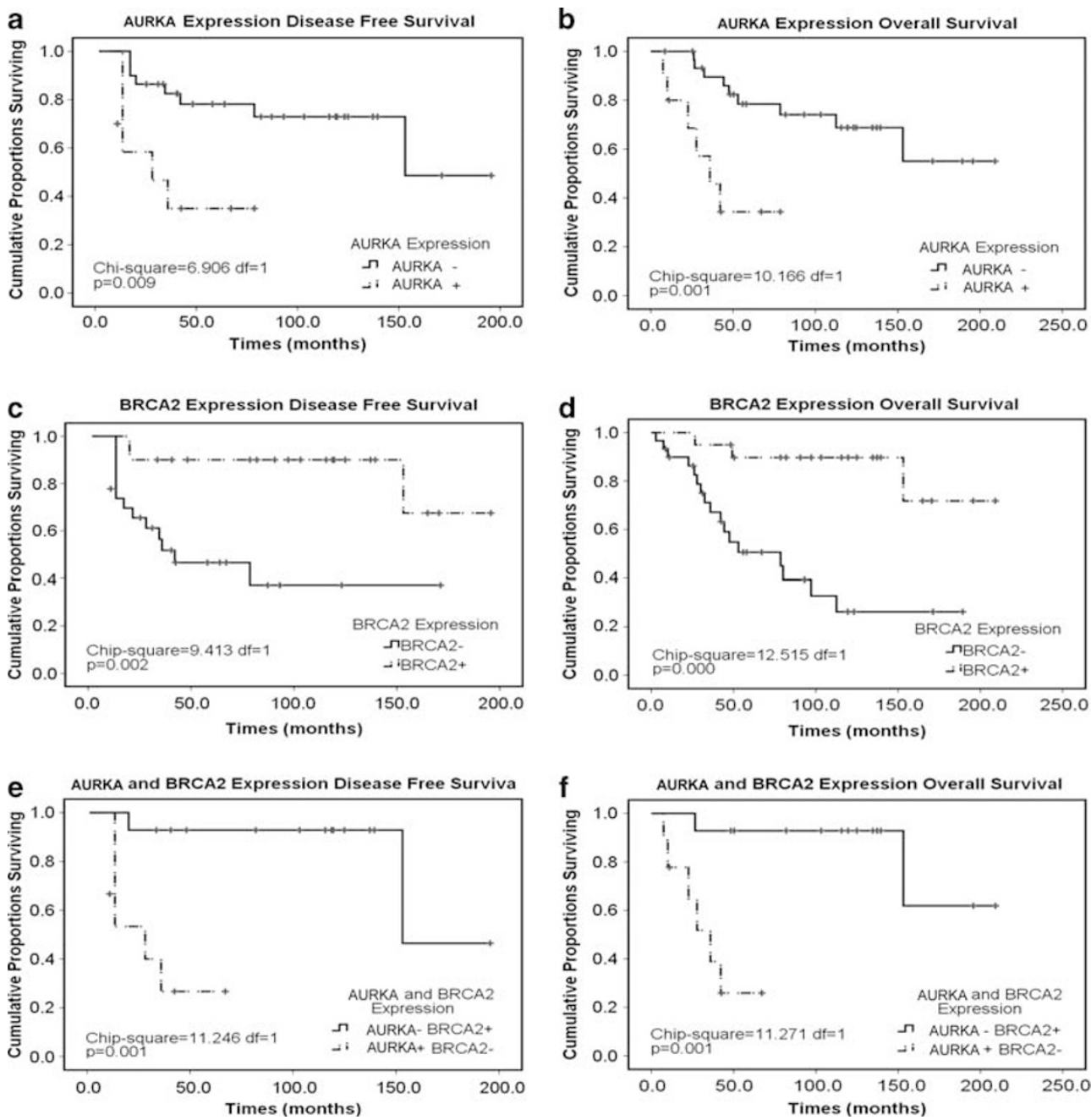

Figure 2 Kaplan-Meier estimates for (a) AURKA disease-free survival, (b) AURKA overall survival, (c) BRCA2 disease-free survival, (d) BRCA2 overall survival, (e) AURKA and BRCA2 disease-free survival, and (f) AURKA and BRCA2 overall survival.

maintenance of DNA damage repair ${ }^{35}$ and genomic integrity. ${ }^{28}$ Inactivation of BRCA2 induces genomic instability as a result of defective DNA damage repair and cell cycle dysregulation in cancer cells. ${ }^{16,33}$ BRCA2 expression was associated with good survival in our study, which demonstrates the tumor suppression function of BRCA2 in endometrioid ovarian carcinoma. Moreover, our results demonstrate a negative correlation between AURKA and BRCA2 expression, which is consistent with the results of a recent report. Yang et $a l^{23}$ found that AURKA knockdown can restore BRCA2 expression, leading to increased genomic stability and decreased tumorigenesis in ovarian cancer cells, suggesting that AURKA negatively regulates BRCA2 expression to control genomic instability. ${ }^{23}$ The reverse correlation between BRCA2 and AURKA expression has also been found in serous ovarian cancer, pancreatic cancer, and breast cancer and thus may represent a general mechanism in epithelial cancers with AURKA overexpression. Yang et $a l^{23}$ found that AURKA overexpression represses p21, pRb, and BRCA2, which promotes cell cycle progression, anti-apoptosis, and genomic instability, leading to increased tumorigenesis. ${ }^{23}$ The clinical correlation between AURKA and BRCA2 expression and patient survival strongly suggests that the negative correlation, illustrated by the results of clinicopathologic and molecular biologic research, can be used to predict endometrioid ovarian carcinoma outcomes.

In summary, our results demonstrate that AURKA expression is an unfavorable prognostic factor in patients with endometrioid ovarian carcinoma, whereas BRCA2 is a favorable prognostic factor. The negative correlation between AURKA and BRCA2 represents a novel prognostic marker for endometrioid ovarian carcinoma.

\section{Acknowledgements}

JL is supported by R01 and an Ovarian SPORE grant (IP50CA83638), and Cancer Center Core grant 
(CA016672) by from the National Cancer Institute and Ovarian Cancer Research Fund Program Developmental Grant (Bast, Overall PI and Liu, PI of pathology core).

\section{Disclosure/conflict of interest}

The authors declare no conflict of interest.

\section{References}

1 Blaustein AKR. Blaustein's Pathology of theFemale Genital Tract, Vol., 4th edn. Springer: New York, 2002.

2 Oral E, Ilvan S, Tustas E, et al. Prevalence of endometriosis in malignant epithelial ovary tumours. Eur J Obstet Gynecol Reprod Biol 2003;109:97-101.

3 Storey DJ, Rush R, Stewart M, et al. Endometrioid epithelial ovarian cancer: 20 years of prospectively collected data from a single center. Cancer 2008;112: 2211-2220.

4 Gilks CB, Ionescu DN, Kalloger SE, et al. Tumor cell type can be reproducibly diagnosed and is of independent prognostic significance in patients with maximally debulked ovarian carcinoma. Hum Pathol 2008;39:1239-1251.

5 Zwart J, Geisler JP, Geisler HE. Five-year survival in patients with endometrioid carcinoma of the ovary versus those with serous carcinoma. Eur J Gynaecol Oncol 1998;19:225-228.

6 Silverberg SG. Histopathologic grading of ovarian carcinoma: a review and proposal. Int J Gynecol Pathol 2000;19:7-15.

7 Hernandez E, Bhagavan BS, Parmley TH, et al. Interobserver variability in the interpretation of epithelial ovarian cancer. Gynecol Oncol 1984;17:117-123.

8 Rosen DG, Zhang Z, Chang B, et al. Low membranous expression of beta-catenin and high mitotic count predict poor prognosis in endometrioid carcinoma of the ovary. Mod Pathol 2010;23:113-122.

9 Glover DM, Leibowitz MH, McLean DA, et al. Mutations in aurora prevent centrosome separation leading to the formation of monopolar spindles. Cell 1995;81: 95-105.

10 Zhou H, Kuang J, Zhong L, et al. Tumour amplified kinase STK15/BTAK induces centrosome amplification, aneuploidy and transformation. Nat Genet 1998; 20:189-193.

11 Stenoien DL, Sen S, Mancini MA, et al. Dynamic association of a tumor amplified kinase, Aurora-A, with the centrosome and mitotic spindle. Cell Motil Cytoskeleton 2003;55:134-146.

12 Nishida N, Nagasaka T, Kashiwagi K, et al. High copy amplification of the Aurora-A gene is associated with chromosomal instability phenotype in human colorectal cancers. Cancer Biol Ther 2007;6:525-533.

13 Sen S, Zhou $\mathrm{H}$, Zhang RD, et al. Amplification/ overexpression of a mitotic kinase gene in human bladder cancer. J Natl Cancer Inst 2002;94:1320-1329.

$14 \mathrm{Li} \mathrm{D}$, Zhu J, Firozi PF, et al. Overexpression of oncogenic STK15/BTAK/Aurora A kinase in human pancreatic cancer. Clin Cancer Res 2003;9:991-997.

15 Sakakura C, Hagiwara A, Yasuoka R, et al. Tumouramplified kinase BTAK is amplified and overexpressed in gastric cancers with possible involvement in aneuploid formation. Br J Cancer 2001;84:824-831.

16 Sen S, Zhou H, White RA. A putative serine/threonine kinase encoding gene BTAK on chromosome 20q13 is amplified and overexpressed in human breast cancer cell lines. Oncogene 1997;14:2195-2200.

$17 \mathrm{Hu} \mathrm{W,} \mathrm{Kavanagh} \mathrm{JJ,} \mathrm{Deaver} \mathrm{M,} \mathrm{et} \mathrm{al.} \mathrm{Frequent} \mathrm{over-}$ expression of STK15/Aurora-A/BTAK and chromosomal instability in tumorigenic cell cultures derived from human ovarian cancer. Oncol Res 2005;15:49-57.

18 Chung CM, Man C, Jin Y, et al. Amplification and overexpression of aurora kinase A (AURKA) in immortalized human ovarian epithelial (HOSE) cells. Mol Carcinog 2005;43:165-174.

19 Gritsko TM, Coppola D, Paciga JE, et al. Activation and overexpression of centrosome kinase BTAK/Aurora-A in human ovarian cancer. Clin Cancer Res 2003;9: 1420-1426.

20 Landen Jr CN, Lin YG, Immaneni A, et al. Overexpression of the centrosomal protein Aurora-A kinase is associated with poor prognosis in epithelial ovarian cancer patients. Clin Cancer Res 2007;13:4098-4104.

21 Lassmann S, Shen Y, Jutting U, et al. Predictive value of Aurora-A/STK15 expression for late stage epithelial ovarian cancer patients treated by adjuvant chemotherapy. Clin Cancer Res 2007;13:4083-4091.

22 Daniels MJ, Wang Y, Lee M, et al. Abnormal cytokinesis in cells deficient in the breast cancer susceptibility protein BRCA2. Science 2004;306:876-879.

23 Yang $\mathrm{G}$, Chang B, Yang $\mathrm{F}$, et al. Aurora kinase A promotes ovarian tumorigenesis through dysregulation of the cell cycle and suppression of BRCA2. Clin Cancer Res 2010;16:3171-3181.

24 Tavassoli FA, Devilee P, (eds.) World Health Organization Classification of Tumours, Pathology and Genetics of Tumours of the Breast and Female Genital Organs. IARC Press: Lyon, 2003;116.

25 Hoskins WJ. Epithelial ovarian carcinoma: principles of primary surgery. Gynecol Oncol 1994;55:S91-S96.

26 Hoskins WJ, McGuire WP, Brady MF, et al. The effect of diameter of largest residual disease on survival after primary cytoreductive surgery in patients with suboptimal residual epithelial ovarian carcinoma. Am J Obstet Gynecol 1994;170:974-979; discussion 9-80.

27 Rosen DG, Yang G, Deavers MT, et al. Cyclin E expression is correlated with tumor progression and predicts a poor prognosis in patients with ovarian carcinoma. Cancer 2006;106:1925-1932.

28 Hirota T, Kunitoku N, Sasayama T, et al. Aurora-A and an interacting activator, the LIM protein Ajuba, are required for mitotic commitment in human cells. Cell 2003;114:585-598.

29 Liu Q, Kaneko S, Yang L, et al. Aurora-A abrogation of p53 DNA binding and transactivation activity by phosphorylation of serine 215. J Biol Chem 2004;279: 52175-52182.

30 Katayama H, Sasai K, Kawai H, et al. Phosphorylation by aurora kinase A induces Mdm2-mediated destabilization and inhibition of p53. Nat Genet 2004;36: $55-62$.

31 Ouchi M, Fujiuchi N, Sasai K, et al. BRCA1 phosphorylation by Aurora-A in the regulation of G2 to $M$ transition. J Biol Chem 2004;279:19643-19648.

32 Zhang Z, Singh M, Davidson S, et al. Activation of BTAK expression in primary ovarian surface epithelial cells of prophylactic ovaries. Mod Pathol 2007;20: 1078-1084. 
33 Tanner MM, Grenman S, Koul A, et al. Frequent amplification of chromosomal region 20q12-q13 in ovarian cancer. Clin Cancer Res 2000;6:1833-1839.

34 Kruk PA, Godwin AK, Hamilton TC, et al. Telomeric instability and reduced proliferative potential in ovarian surface epithelial cells from women with a family history of ovarian cancer. Gynecol Oncol 1999; 73:229-236.

35 He QY, Zhou Y, Wong E, et al. Proteomic analysis of a preneoplastic phenotype in ovarian surface epithelial cells derived from prophylactic oophorectomies. Gynecol Oncol 2005;98:68-76. 